Supporting Information for

\title{
Relationship between Mechanical and Electrical Properties of Continuous \\ Polymer-Free Carbon Nanotube Fibers by Wet-Spinning Method and \\ Nanotube-Length Estimated by Far-Infrared Spectroscopy Spectroscopy
}

Xueli $\mathrm{Wu}^{\dagger}$, Takahiro Morimoto ${ }^{\dagger, \ddagger}, K_{\text {Ken Mukai }}{ }^{\dagger, \neq, \S}$, Kinji Asaka ${ }^{\dagger, \neq, \S}$, Toshiya Okazaki ${ }^{*,+, \neq}$

${ }^{\dagger}$ Technology Research Association for Single Wall Carbon Nanotubes (TASC), Tsukuba 305-

8565, Japan

${ }^{\ddagger}$ CNT-Application Research Center, National Institute of Advanced Industrial Science and

Technology (AIST), Tsukuba 305-8565, Japan

${ }^{\S}$ Inorganic Functional Materials Research Institute, National Institute of Advanced Industrial

Science and Technology (AIST), Osaka, 563-8577, Japan

*Corresponding author: Tel: +81-29-861-4173 E-mail: toshi.okazaki@aist.go.jp 


\section{SG-CNT Suspensions with SG-CNTs Concentration of 0.20 wt\% (0.20 wt \% SG-CNT)}

Injection Images of 0.20 wt\% SG-CNT Fibers. Figure S1 shows the injection images of SGCNT suspensions at a concentration of $0.20 \mathrm{wt} \%$ (0.20 wt\% SG-CNT) dispersed with an ultrasonic probe from 10 to 60 min and each fiber's SEM images. Similar to that of $0.28 \mathrm{wt} \%$ SG-CNT, the SG-CNT suspensions dispersed for 60 min did not form good fiber.

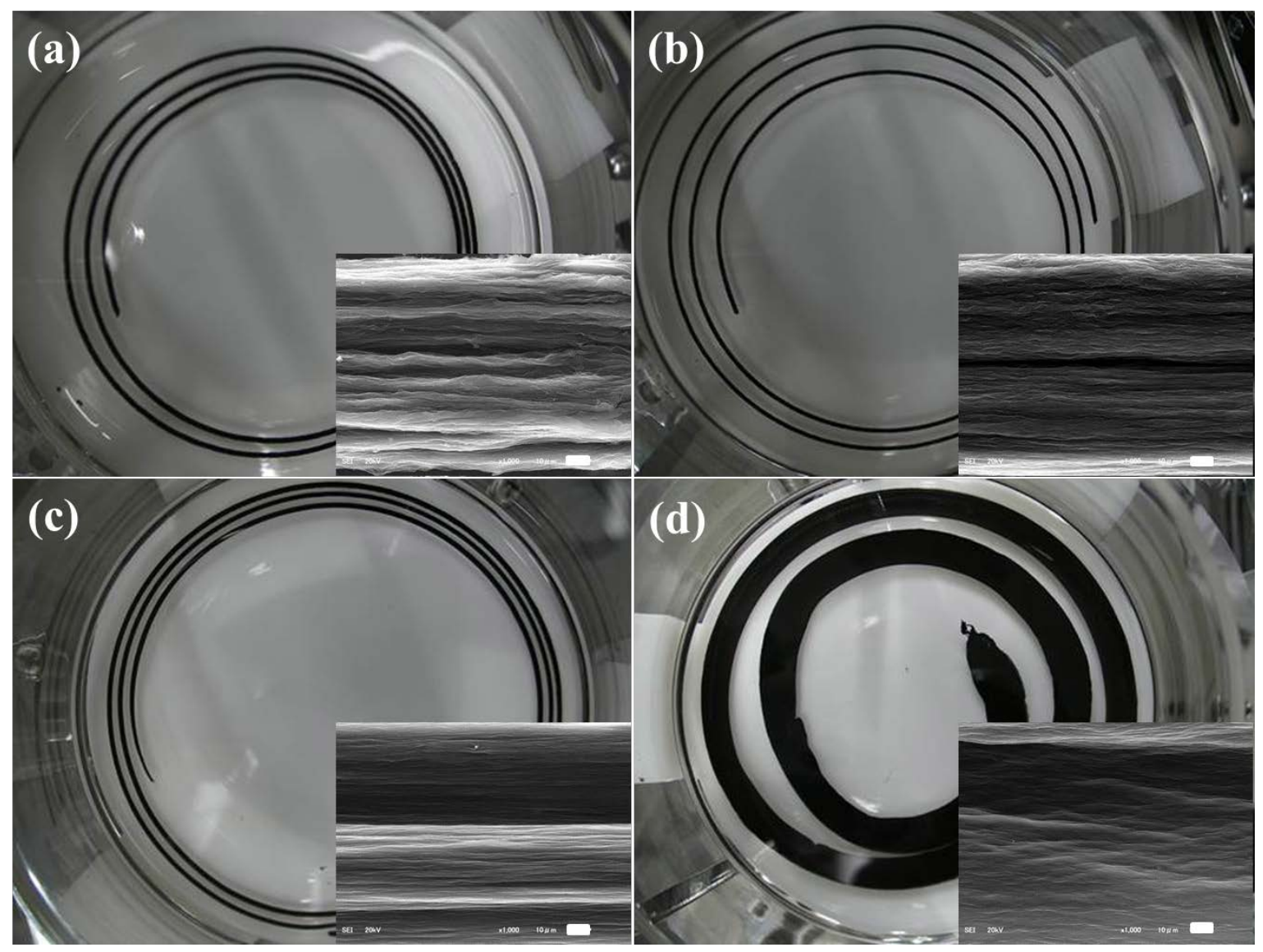

Figure S1. Injection images of 0.20 wt\% SG-CNT suspensions into IPA with different dispersing times of (a) 10, (b) 20, (c) 30, and (d) 60 min (Inset: SEM photographs of each CNT fiber, scale bar=10 $\mu \mathrm{m})$.

0.20 wt\% SG-CNT Fibers Properties. Figure S2 shows (a) Young's moduli, (b) fracture strengths, and (c) electric conductivities of $0.20 \mathrm{wt} \%$ SG-CNT fibers with various sonication times. All were highest when the suspension was dispersed for $30 \mathrm{~min}$, which is also consistent with the results of $0.28 \mathrm{wt} \%$ SG-CNT fibers. 

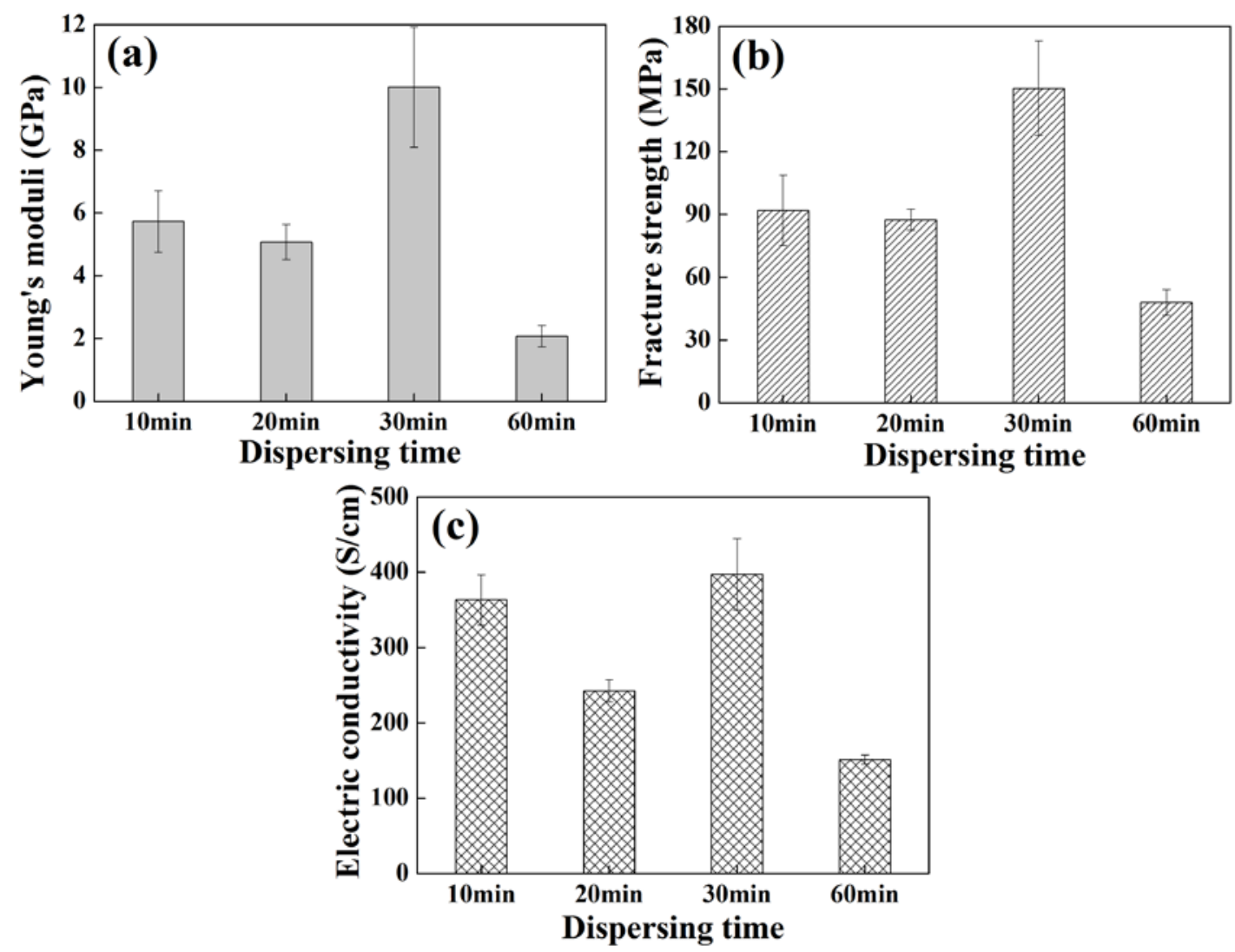

Figure S2. Mechanical and electrical properties of 0.20 wt\% SG-CNT fibers: (a) Young’s moduli, (b) fracture strengths, and (c) electric conductivities.

FIR and Raman Spectra of 0.20 wt\% SG-CNT Suspensions. The characterization of $0.20 \mathrm{wt} \%$ SG-CNT suspensions was conducted by resonance Raman and FIR spectroscopy. Figure S3 shows (a) Raman spectra and (b) FIR spectra of $0.20 \mathrm{wt} \%$ SG-CNT suspensions with different dispersing times. The G/D values are 2.2, 2.0, 2.6, and 2.4 for 10, 20, 30, and $60 \mathrm{~min}$, respectively. The absence of any significant change of the G/D values suggests that the ultrasonic dispersing did not severely affect the surface disorder of SG-CNTs. The peak positions were at approximately 302, 367, 290, and $346 \mathrm{~cm}^{-1}$ for 10, 20, 30, and $60 \mathrm{~min}$, respectively. The FIR peak position of the CNT suspensions dispersed for 30 min was lower than those of the others, indicating that the CNTs in the suspensions were longer. The better fiber properties of the 30 min-processed sample indicate that a damage-free dispersion process of CNTs is very important for preparing high performance CNT fibers. 

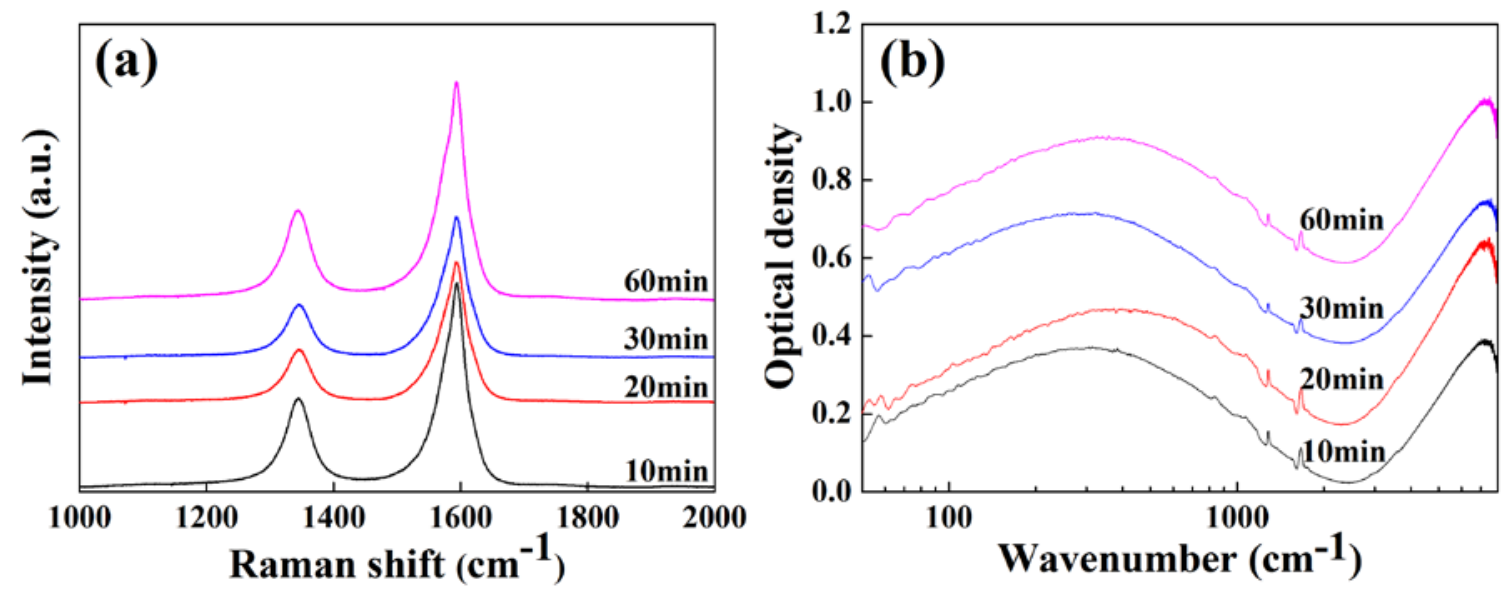

Figure S3. (a) Resonance Raman and (b) FIR spectra of 0.20 wt\% SG-CNT suspensions with different dispersing times. 